\title{
Fabrication of Protein Microarrays for Immunoassay Using the Electrospray Deposition (ESD) Method
}

\author{
BUMHWAN LEE ${ }^{1}$, JOONWAN KIM ${ }^{2}$, \\ KAZUHISA ISHIMOTO ${ }^{1}$, YUTAKA YAMAGATA ${ }^{2}$, \\ AKIHIKO TANIOKA ${ }^{3}$ AND TERUYUKI NAGAMUNE ${ }^{1}$ \\ ${ }^{1}$ Department of Chemistry and Biotechnology, \\ School of Engineering, The University of Tokyo, \\ Tokyo113-8656, Japan \\ ${ }^{2}$ The Institute of Physical and Chemical Research (RIKEN), \\ Materials Fabrication Laboratory, Wako 351-0198, Japan \\ ${ }^{3}$ Department of Organic and Polymeric Materials, \\ International Research Center of Macromolecular Science, \\ Tokyo Institute of Technology, Tokyo152-8552, Japan
}

Keywords: Electrospray Deposition, Protein Array, Immunoassay

In recent years new methods for active protein film fabrication have been rapidly developing. One of these methods is the electrospray deposition (ESD) method. This method allows the spontaneous deposition of many identical dots and has a remarkable spatial resolution as well as the ability to overcome limitations like low rates of deposition, low efficiency of substance transfer, and cross-contamination. In this study, an antibody-based protein array for high-throughput immunoassay was fabricated with the ESD method using a quartz mask with holes made by an abrasive jet technique. An antibody solution was electrosprayed onto an ITO glass and then deposited antibodies were cross-linked with a vapor of glutaraldehyde. The diameter of the spots was approximately $150 \mu \mathrm{m}$. The arrays were then incubated with corresponding target antigenic molecules and washed. The captured antigens were collectively detected by fluorescence and chemiluminescence. These signals were quantitatively visualized with a highresolution charge-coupled device $(\mathrm{CCD})$ detector. Sensitive $(\sim 1 \mathrm{ng} / \mathrm{m} l)$ and simultaneous detection of various antigens could be performed by enzyme linked immunosorbent assay or fluorescence immunoassay using this system.

\section{Introduction}

In recent years, the complete sequencing of the human genome has allowed the analysis of gene expression. With gene expression profiling, DNA arrays containing multicomponent microarrays have been reported (Schena et al., 1995; Chee et al., 1996), thus providing information regarding molecular assays, such as, differential gene expression and DNA sequencing. Although this information plays an important role, there are some limitations in the information that the DNA arrays can provide. DNA arrays cannot, for example, provide protein level information such as that concerning the protein-DNA interaction, post-translational modifications and protein-protein interactions, which are very important in cell functions. It is necessary therefore, to develop a protein-based microarray to study protein functions.

To develop these protein arrays, it is important to immobilize proteins on a support in a way that pre-

Received on February 3, 2003. Correspondence concerning this article should be addressed to T. Nagamune (E-mail address: nagamune@bio.t.u-tokyo.ac.jp). serves their functional activities. There have been a number of studies focusing on the deposition of protein and other biomolecules in the fields of genomic, proteomic, clinical and pharmaceutical analyses. Methods enabling active protein film formation are rapidly developing and there are now many different types. For example, proteins have been deposited using photolithography and lift-off techniques (Pritchard et al., 1995; Mooney et al., 1996), a robotic micromanipulation system including ink jetting (Newman et al., 1992), a stamp microcontact printing method (Bernard et al., 1998), and electrospray deposition techniques (Morozov and Morozova, 1999). Among these methods, the electrospray deposition (ESD) method is the most significant as it allows the spontaneous deposition of many identical dots, it has a remarkable spatial resolution and it overcomes limitations like low rates of deposition, low efficiency of substance transfer, and cross-contamination.

In the ESD method, solutions of a substance are electrosprayed and charged particles are deposited onto specified areas of the conductive substrates under the control of electrostatic forces. The ESD method was first used to fabricate thin radioactive sources in nu- 
clear physics. Other known applications include the formation of layers of semiconductive ceramics like metal oxide films (Chen et al., 1996), the formation of protective polymer coatings on electrode surfaces (Hoyer et al., 1996), the modification of silicon surfaces with layers of silk-forming peptides to enhance the adhesion of living cells, the preparation of DNA and protein samples for scanning tunneling microscopy (STM) (Thundat et al., 1992), and ionization in mass spectrometry (MS) (Matsuo et al., 1979).

In the ESD of proteins, however, the main problem is preserving the functional properties of these molecules upon electrospray and the subsequent impact of the charged electrospray (ES) products with the substrate surface. Under proper conditions the ESdeposited proteins are known to have preserved their enzymatic activities and to have been recognized specifically by antibodies (Morozov and Morozova, 1999).

In this study, we fabricate a protein multimicroarray with several kinds of anti-immunoglobulin (IgG) antibodies using the electrospray deposition (ESD) technique. The feasibility of simultaneous and specified detection methods for numerous IgGs as antigens using a microarray-based enzyme linked immunosorbent assay (ELISA) and fluorescence immunoassay (FIA) format is also demonstrated.

\section{Materials and Methods}

\subsection{Materials}

Antigens (mouse, human, bovine, chicken, rabbit and guinea pig IgGs) and their corresponding anti-IgG antibodies were purchased from the Sigma Chemical Company and Jackson Immuno Research. ECL substrate was purchased from Amersham. Other chemicals were purchased from the Sigma Chemical Company.

\subsection{The ESD device}

The ESD device is schematically illustrated in Fig. 1. The deposition of protein was performed under the control of a local electrostatic field, which attracts charged ES products to specified substrate areas. By masking a conducting substrate with a dielectric mask with holes, which is covered with Teflon-coated copper film, a local electric field was formed between the capillary and the substrate. Protein was then deposited on the substrate through the holes in the mask. To improve the efficiency of the spraying, a collimating voltage was applied to the thin copper film between the surface of the mask and the Teflon film. A glass capillary with internal platinum electrodes of $50 \mu \mathrm{m}$ in diameter and a flat dielectric mask with holes of $300 \mu \mathrm{m}$ in diameter were used (Fig. 3(a)). The holes of the mask were fabricated using an abrasive jet technique (MB1ML-004, SINTOBRATOR, Aichi, Japan), which is utilized as the fabrication method of quartz masks. This process is graphically indicated in Fig. 2. First, a trans-

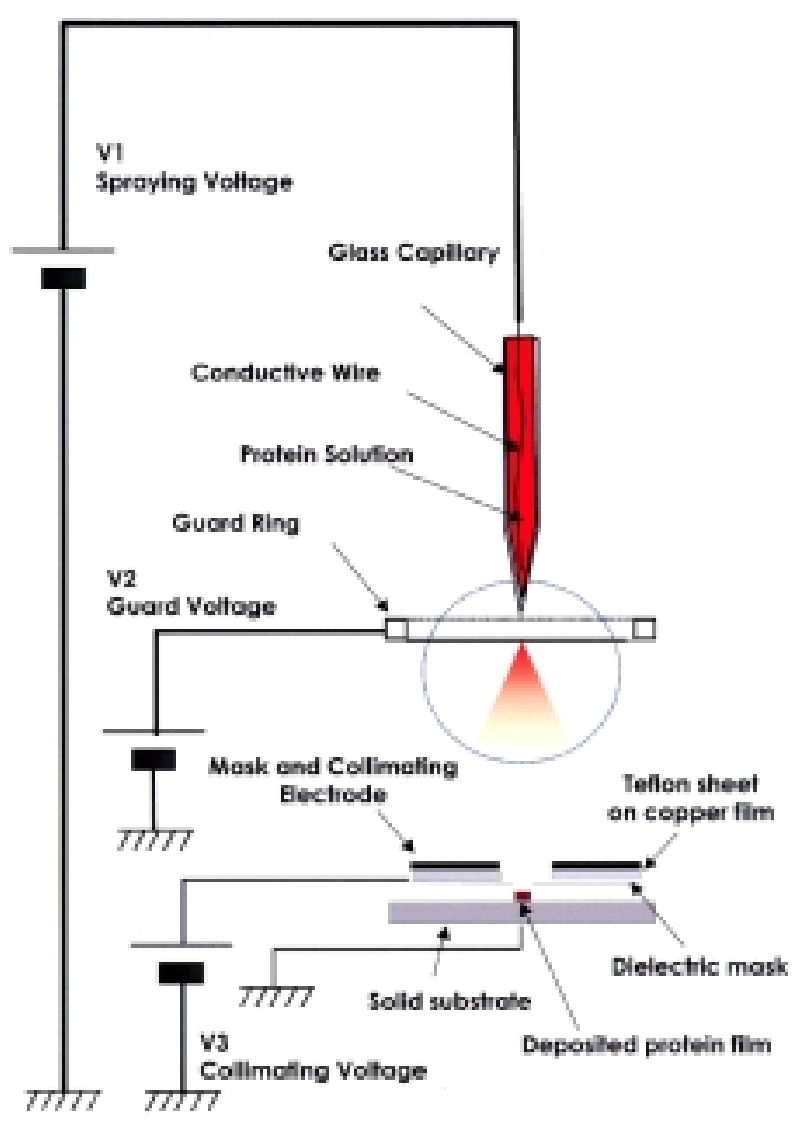

Fig. 1 Schematic diagram of the electrospray deposition device

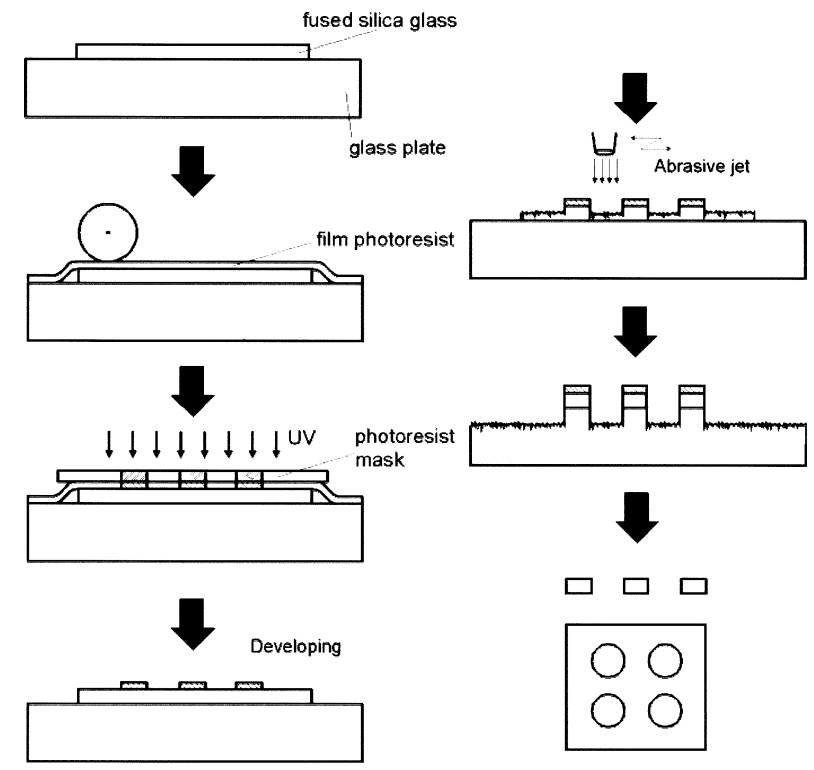

Fig. 2 Fabrication flow diagram of the mask

parent glass plate coated with thin ITO $(20 \Omega / s q)$ was used as a substrate. The device was then placed in a plastic chamber to control humidity and protect against the deposition of dust particles present in the ambient 

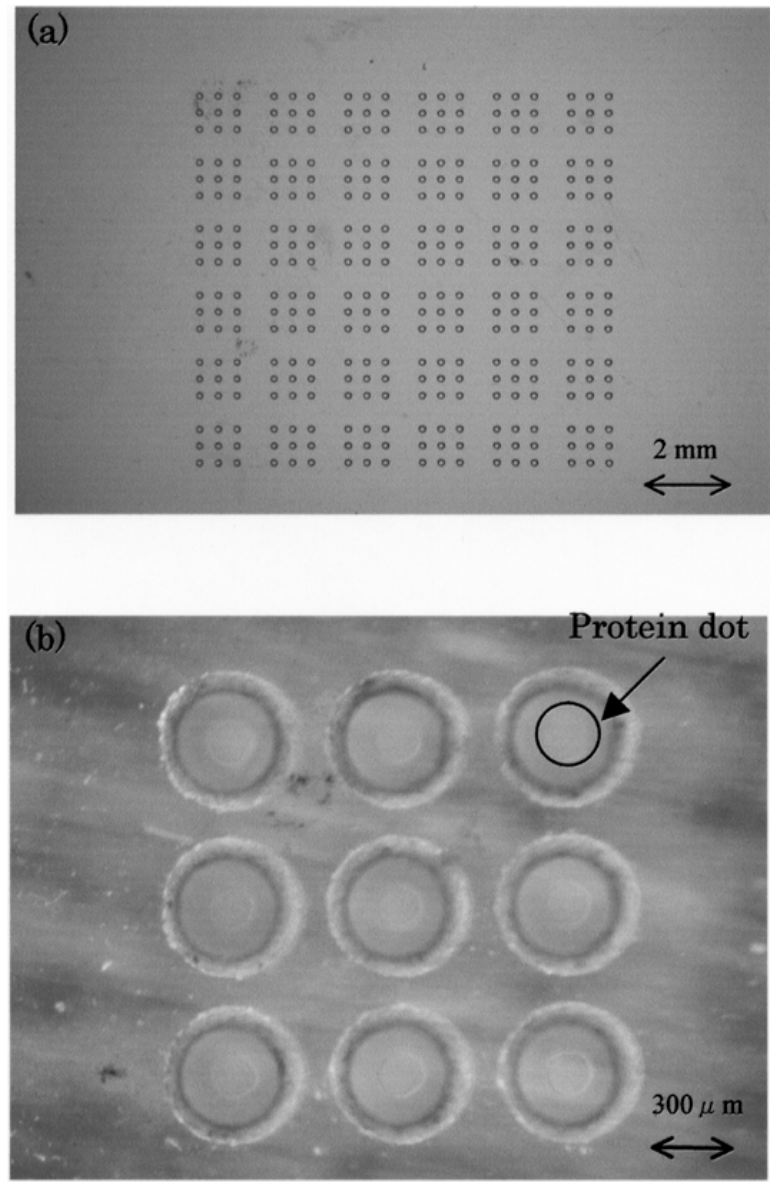

Fig. 3 Image of mask and electrospray deposited protein spots: (a) mask with 324 holes, (b) protein deposited on substrate using the mask

air. Humidity was controlled to below $30 \%$ by pumping filtered dry air into the chamber and by using desiccants. The ES plume was illuminated with a beam from a He-Ne laser.

\subsection{Preparation of the protein array}

The anti-IgG antibody solutions, desalted using a PD-10 column to reduce their electronic conductivity to less than $500 \mu \mathrm{S} / \mathrm{cm}$, were ES-deposited and crosslinked with glutaraldehyde vapor. Typical electrospraying conditions for the deposition of proteins were: voltage $3.0-4.0 \mathrm{kV}$, humidity $20-30 \%$. Slight pressure was applied to the protein solution in the glass capillary to increase the spray rate from the nozzle. Surface analysis of the microarray was performed by atomic force microscopy (AFM) (Nanopics 1000, Seiko Instrument, Chiba, Japan) and field emission gun scanning electro microscopy (FE-SEM) (S800, Hitachi, Tokyo, Japan).

\subsection{Immunoassay with antibody microarray}

The anti-IgG antibody array plates were blocked with $2 \%$ skimmed milk in PBS for $2 \mathrm{~h}$ at room temperature. After blocking, the plates were incubated with appropriate quantities of HRP or FITC conjugated $\operatorname{IgG}$
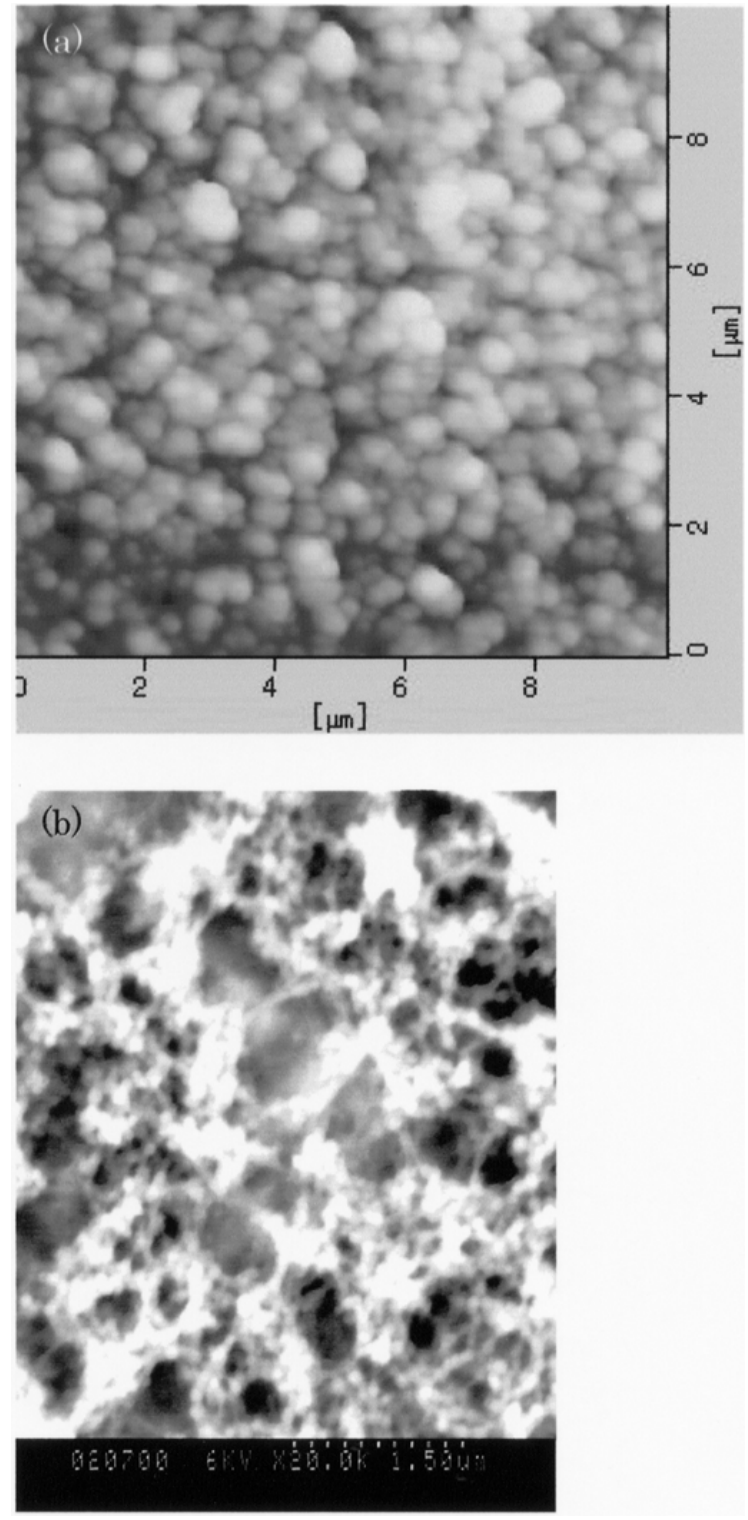

Fig. 4 AFM and SEM images of a deposited protein spot: (a) AFM image, (b) SEM image of the inside of a spot

antigens $(100 \mathrm{ng} / \mathrm{ml})$ for $1 \mathrm{~h}$ at $37^{\circ} \mathrm{C}$. Excess antigens were then removed by washing the plate five times with PBS $/ 0.1 \%$ Tween 20 (5 min each) and five times with PBS (5 min each).

For ELISA detection, the plates capturing the HRP conjugated IgGs were incubated with ECL substrates according to the manufacturer's instructions. The plates were placed on a sheet of X-ray film (X-OMAT, Fujifilm, Tokyo, Japan) for development by autoradiography and also imaged with a digital CCD camera coupled with image intensifier (C2400-35, Hamamatsu, Shizuoka, Japan). For FIA detection, the plates capturing FITC conjugated mouse IgGs were placed on a quartz glass and imaged using a CCD camera system. The intensities of chemiluminescence and the fluores- 

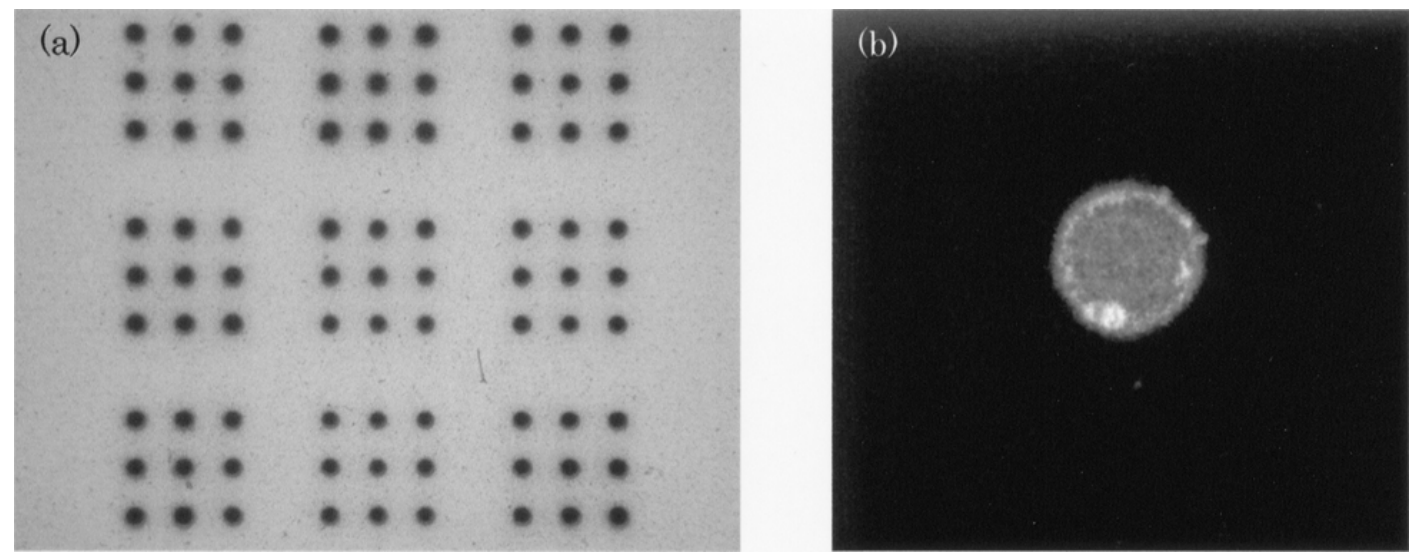

Fig. 5 Detection of mouse IgG using an anti-mouse IgG microarray fabricated using the ESD method: (a) detection with $\mathrm{X}$-ray film, (b) detection with a digital CCD camera

cence of the spots were measured using an image analysis system (ARGUS-20, Hamamatsu, Shizuoka, Japan).

\section{Results and Discussion}

\subsection{The fabrication of antibody microarray by the ESD method and investigation with SEM and AFM}

To fabricate the multi-component microarray, a mask was produced using an abrasive jet method. Using this mask, a solution of anti-mouse $\mathrm{IgG}$ was electrosprayed onto an ITO substrate under the conditions mentioned in the experimental section. The deposited spots are shown in Fig. 3(b), they were semitransparent and approximately $150 \mu \mathrm{m}$ in diameter.

To investigate the uniformity of the deposited spots, the surface was imaged with AFM and FE-SEM. The diameter of the cluster was smaller than $400 \mathrm{~nm}$ and almost uniform on a microscopic scale level (Fig. 4(a)). The inside structures were observed using SEM. Results showed that the spots have porous membrane structures.

\subsection{ELISA with antibody microarray}

To develop a sensitive and specific method for simultaneous multiple antigen detection, the enhanced chemiluminescence (ECL) detection method and the specificity of ELISA were used.

First, the activity of the electrosprayed proteins was investigated. The light generated in the oxidization of luminol by HRP was detected and visualized by exposure to X-ray film. A digital CCD camera coupled with an image intensifier was also used to detect the light signals. As shown in Fig. 5, signals were detected using both methods. Next, anti-mouse IgG and $\alpha$-lactalbumin were electrosprayed onto an ITO plate and exposed to the ELISA process with HRP-conjugated mouse $\operatorname{IgG}(100 \mathrm{ng} / \mathrm{ml})$ to test the non-specific adsorption of mouse IgG to the microarray. As shown

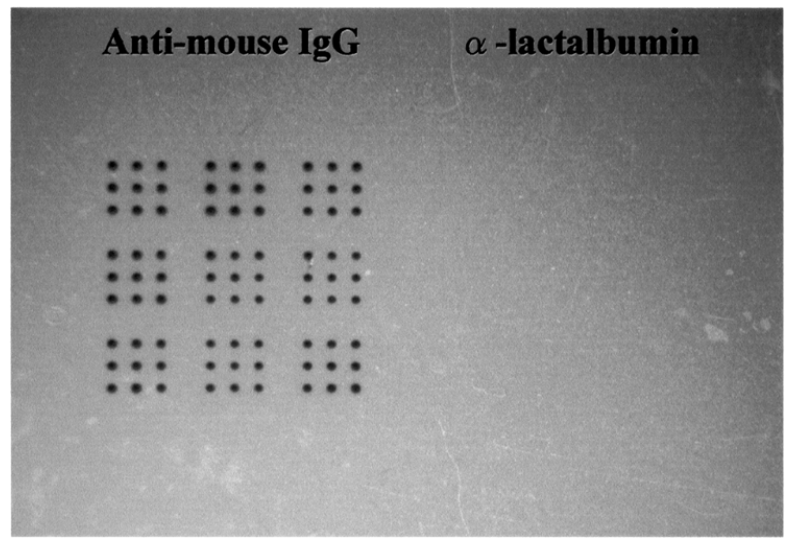

Fig. 6 Detection of mouse IgG. The spots of deposited $\alpha$-lactalbumin showed no signal against mouse IgG conjugated with HRP

in Fig. 6, no signals were detected for $\alpha$-lactalbumin, specific signals for anti-mouse IgG only were detected. These results indicate that the anti-mouse $\mathrm{IgG}$ microarray fabricated by the ESD method and crosslinking with glutaraldehyde preserves immunological activity.

2.3 ELISA with a multi-component anti-IgG antibody microarray fabricated by the ESD method

The multi anti-IgG antibodies deposited microarray, which recognizes IgGs from six kinds of animals (mouse, human, bovine, chicken, rabbit and guinea pig), was fabricated. After the blocking step, the array was incubated with solutions containing cognate HRP conjugated $\mathrm{IgG}(100 \mathrm{ng} / \mathrm{ml})$ in PBS (with $2 \%$ skimmed milk) to demonstrate the specificity of each deposited antibody spot to the cognate antigen. The signals of each antigen-antibody complex were detected using X-ray film as shown in Fig. 7. Specific signals were only observed between corresponding antigens (IgGs) and antibodies (cognate anti-IgGs). 



Fig. 7 Detection of IgGs refined from six kinds of animals. A non-specific signal was not observed: (a) mouse, (b) human, (c) bovine, (d) chicken, (e) rabbit, (f) guinea pig
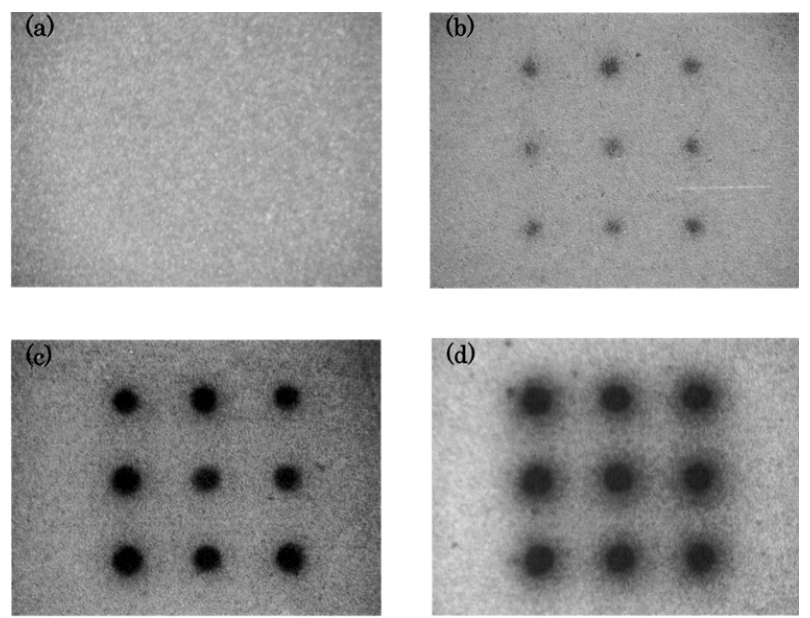

Fig. 8 The sensitivity of ELISA in the detection of HRP conjugated mouse IgG using the ECL method: (a) $0.1 \mathrm{ng} / \mathrm{ml}$, (b) $1 \mathrm{ng} / \mathrm{ml}$, (c) $100 \mathrm{ng} / \mathrm{ml}$, (d) $1 \mu \mathrm{g} /$ $\mathrm{m} l$ of mouse $\mathrm{IgG}$

These results further imply that multi protein microarrays fabricated by the ESD method and crosslinking with glutaraldehyde vapor can be applicable in the detection of protein from biological samples.

\subsection{Sensitivities of ELISA and FIA using antibody microarray fabricated by the ESD method}

The sensitivity of ELISA using anti-mouse IgG microarray plates fabricated with electrospray, were examined. Following the blocking procedure, the plates were incubated with different concentrations of HRPconjugated mouse IgG. As shown in Fig. 8, the levels of antigen as low as $1 \mathrm{ng} / \mathrm{m} l$ could be detected using the ECL method and X-ray film. Next, the sensitivity of FIA using the microarray was also examined. Antimouse IgG was deposited and immobilized onto an ITO glass. After the blocking process, the plate was placed on skimmed milk solution ( $2 \%$ ) with different concentrations of mouse IgG conjugated with FITC as the antigen. After washing, the fluorescent signal of the antigen-antibody complex was detected by a digital CCD camera. As shown in Fig. 9, levels of mouse IgG lower than $1 \mathrm{ng} / \mathrm{ml}$ could be detected.

\section{Conclusion}

This study served to fabricate antibody microarrays using the ESD method. Furthermore, the feasibility of this system in the sensitive detection of antigens with the multiplexed ELISA format, compatible with high-throughput analysis systems, was also evaluated. The results shown here suggest that this system could be applicable in high-throughput protein detection systems. The ESD method is very efficient at depositing protein, more than 324 spots can be fabricated in $20 \mathrm{~min}$. These electrosprayed protein spots were also active and very uniform, which is advantageous in the reproducible quantification of antigens. The chip based multi-microarray fabricated by the ESD method showed neither non-specific adsorption nor 

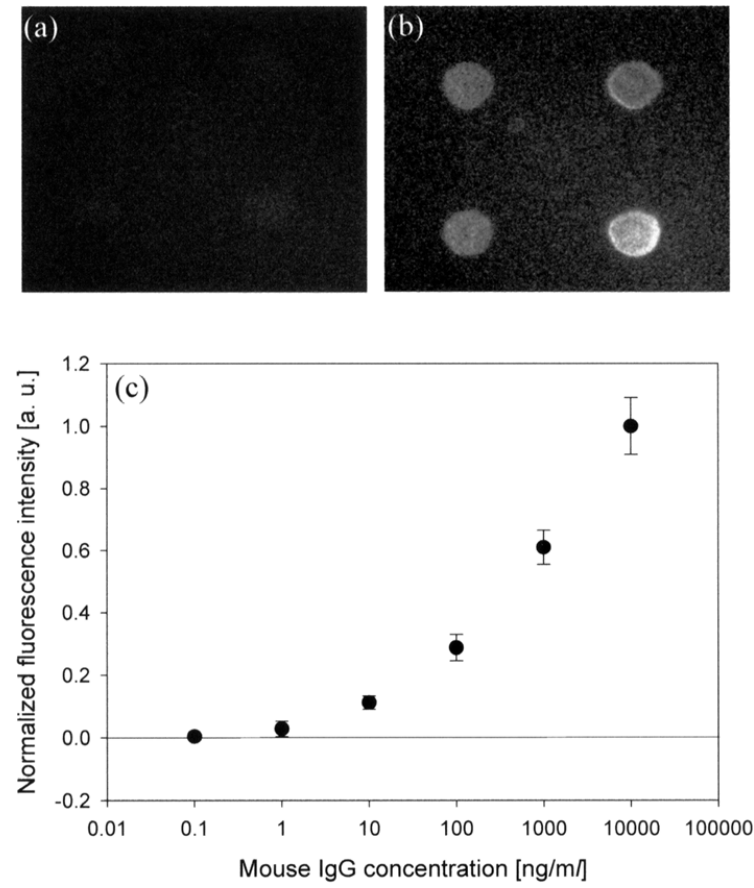

Fig. 9 The sensitivity of FIA in the detection of FITC conjugated mouse $\mathrm{IgG}$ : (a) $0.1 \mathrm{ng} / \mathrm{ml}$, (b) $100 \mathrm{ng} / \mathrm{ml}$, (c) standard curve (normalized fluorescent intensity vs. mouse $\mathrm{IgG}$ concentration)

non-specific binding. In this study sensitivity reached as low as $1 \mathrm{ng} / \mathrm{ml}$, however, lower detection limits may be possible as these preliminary microarray systems were not designed for maximum assay sensitivity. Methods for improving protein immobilization on ITO plates and increasing assay sensitivity are under way.

\section{Acknowledgment}

This study was supported in part by a Grant-in-Aid for Scientific Research on Priority Areas (296-10145107) from the Ministry of Education, Science, Sports and Culture of Japan.

\section{Literature Cited}

Bernard, A., E. Delamarche, H. Schmid, B. Michel, H. R. Bosshard and H. Biebuyck; "Printing Patterns of Proteins," Langmuir, 14, 2225-2229 (1998)

Chee, M., R. Yang, E. Hubbell, A. Berno, X. C. Huang, D. Stern, J. Winkler, D. J. Lockhart, M. S. Morris and S. P. A. Fodor; "Accessing Genetic Information with High-Density DNA Arrays," Science, 274, 610-614 (1996)

Chen, C. H., E. M. Kelder, P. J. J. M. van der Put and J. Schoonman; "Morphology Control of Thin $\mathrm{LiCoO}_{2}$ Films Fabricated Using the Electrostatic Spray Deposition (ESD) Technique," J. Mater. Chem., 6, 765-771 (1996)

Hoyer, B., G. Sorensen, N. Jensen, D. B. Nielsen and B. Larsen; "Electrostatic Spraying: A Novel Technique for Preparation of Polymer Coatings on Electrodes," Anal. Chem., 68, 3840-3844 (1996)

Matsuo, T., H. Matsuda and I. Katakuse; "Silicon Emitter for Field Desorption Mass-Spectrometry," Anal. Chem., 51, 1329-1331 (1979)

Mooney, J. F., A. J. Hunt, J. R. McIntosh, C. A. Liberko, D. M. Walba and C. T. Rogers; "Patterning of Functional Antibodies and Other Proteins by Photolithography of Silane Monolayers," Proc. Natl. Acad. Sci. U.S.A., 93, 12287-12291 (1996)

Morozov, V. N. and T. Y. Morozova; "Electrospray Deposition as a Method to Fabricate Functionally Active Protein Films," Anal. Chem., 71, 1415-1420 (1999)

Newman, J. D., A. P. F. Turner and G. Marrazza; "Ink-jet Printing for the Fabrication of Amperometric Glucose Biosensors," Anal. Chim. Acta., 262, 13-17 (1992)

Pritchard, D. J., H. Morgan and J. M. Cooper; "Patterning and Regeneration of Surfaces with Antibodies," Anal. Chem., 67, 36053607 (1995)

Schena, M., D. Shalon, R. W. Davis and P. O. Brown; "Quantitative Monitoring of Gene-Expression Patterns with a Complementary-DNA Microarray," Science, 270, 467-470 (1995)

Thundat, T., R. J. Warmack, D. P. Allison and T. L. Ferrel; "Electrostatic Spraying of DNA-Molecules for Investigation by Scanning Tunneling Microscopy," Ultramicroscopy, 42, 1083-1087 (1992) 